\title{
Partition Behavior of $p$-Aminobenzoic Acid and Sulfonamides at Various pH Values
}

\author{
Hiroshi Terada \\ Faculty of Pharmacentical Sciences, University of Tokushima1)
}

(Received September 20, 1971)

\begin{abstract}
The partition behaviors of amphoteric acids with $\mathrm{pH}$ were examined based on the theoretical considerations. The apparent partition coefficient, defined as the ratio of the activities of the acid in organic and aqueous phases, is maximal at the isoelectric point. When $\mathrm{p} K_{1}$ is much smaller than $\mathrm{p} K_{2}$, the apparent partition coefficient coincides with the true partition coefficient, defined as the ratio of the activities of the neutral form of the acid in the two phases, at this point. The former changes linearly with slopes of +1 and -1 in the region of $\mathrm{p} K_{1} \gg \mathrm{pH}$ and $\mathrm{p} K_{2} \ll \mathrm{pH}$. By applying the method of Ang ${ }^{2}$ when the presence of the zwitter-ionic form can be excluded, it was demonstrated that the true partition coefficient can be determined exactly from this $\mathrm{pH}$-partition curve, even if the ionization constants of the acid are not known. This method also gives the values of the two ionization constants, so it seemed to be available for determination of the ionization constants of acids, which are very slightly soluble in water.

The partition coefficients of $p$-aminobenzoic acid, sulfonamide and sulfamonomethoxine were measured in an $n$-octanol $/ \mathrm{H}_{2} \mathrm{O}$ system at $25^{\circ}$ over a wide $\mathrm{pH}$ range. The variation of the partition coefficients with $\mathrm{pH}$ confirmed the above considerations well. The values obtained for the true partition coefficients and ionization constants agreed well with the results of others.
\end{abstract}

It is observed in many cases that increase in the hydrophobic nature of organic compounds results in increased membrane permeability ${ }^{3)}$ and greater biological activity. ${ }^{4)}$ This is considered to be because biomembranes are hydrophobic and the sites of action of drugs are mostly in membranes. Therefore, organic solvents are often used as models of biomembranes in studies on membrane permeability ${ }^{5}$ or structure-activity relationships. ${ }^{6}$ ) The hydrophobicity of a compound can be estimated from apparent or true partition coefficient. The true partition coefficient is defined as the ratio of the activity of the neutral form of a compound in an organic solvent to that in water and it is governed by the physicochemical conditions of the partition system. Thus for the above purpose this is preferable to the apparent partition coefficient, as pointed out by Fujita, et al. ${ }^{7)}$ However, it is often difficult to estimate the true partition coefficient of an organic compound, since it may be present in several forms in either or both phases due to dissociation or association between molecules (nonionizednonionized $^{8}$ ) or nonionized-ionized ${ }^{9}$ ) or hydration ${ }^{10}$.) Furthermore among drugs, many organic acids have two or more ionizable groups. Their partition coefficients have not always

1) Location: Shomachi-1, Tokushima.

2) K. Ang, J. Phys. Chem., 62, 1109 (1958).

3) L.S. Schanker, P.A. Nafpliotis, and J.M. Johnson, J. Pharmacol. Exptl. Therap., 133, 325 (1961); L.B. Holder and S.L. Hayes, Mol. Pharmacol., 1, 266 (1965).

4) A. Albert, "Selective Toxicity," Methuen, London, 1968, p. 436.

5) H.L. Rosano, K. Breindel, J.H. Shulman, and A.J. Eydt, J. Colloid Interface Sci., 22, 58 (1966); H.P. Ting, G.L. Bertrand, and D.F. Sears, Biophys. J., 6, 813 (1966).

6) C. Hansch, "Physicochemical Aspects of Drug Action," ed. by E.J. Ariëns, Pergamon, London, 1966, pp. $141-167$.

7) T. Fujita, J. Iwasa, and C. Hansch, J. Am. Chem. Soc., 86, 5175 (1964).

8) D.S. Goodman, J. Am. Chem. Soc., 80, 3887 (1958).

9) A. Finkelstein, Biochim. Biophys. Acta, 205, 1 (1970).

10) R. Van Duyne, S. Taylor, S. Christian, and H. Affsprung, J. Phys. Chem., 71, 3427 (1967). 
been determined exactly, possibly partly because it has been difficult to determine their ionization constants due to their low solubilities. Accordingly we studied the distribution of some amphoteric acids ( $p$-aminobenzoic acid, sulfonamide and sulfamonomethoxine) between $n$-octanol and water to obtain fundamental information on the mechanism of their distribution between the aqueous and hydrophobic phases. We also developed a method for determination of the exact values of the true partition coefficients of acids when their ionization constants are not known. Application of this method for determination of the ionization constants of amphoteric acids was also examined with good results.

\section{Result and Discussion}

\section{Determination of True Partition Coefficients}

Assuming that a certain amphoteric acid, which takes cationic, neutral, zwitter-ionic and anionic forms by dissociation in $\mathrm{H}_{2} \mathrm{O}$, is distributed between organic and aqueous phases in the manner shown in Chart 1 , the apparent partition coefficient $(Q)$ can be represented by Eq. (1). In Chart 1, the effects of dissociation and hydration of molecules in the organic phase and association between molecules in both phases on the distribution are not taken into account. (+AO), (OAO), (+A-) and (OA-) denote the activities of the cationic, neutral, zwitter-ionic and anionic forms of the acid $(A)$ and the subscripts $w$ and $o$ indicate the aqueous and organic phases, respectively. $K_{a}, K_{b}, K_{c}$, and $K_{d}$ are microscopic acid

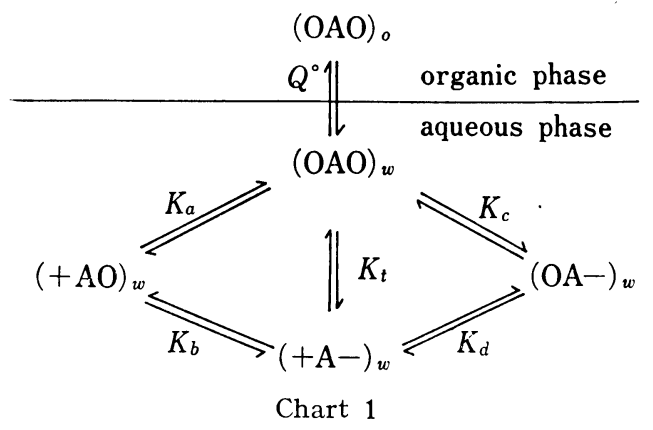
dissociation constants in each equilibrium

$$
Q=\frac{(\mathrm{OAO})_{o}}{(+\mathrm{AO})_{w}+(\mathrm{OAO})_{w}+(+\mathrm{A}-)_{w}+(\mathrm{OA}-)_{w}}
$$

and the relationships between them are as follows:

$$
\begin{aligned}
& K_{1}=K_{a}+K_{b} \\
& 1 / K_{2}=1 / K_{c}+1 / K_{d} \\
& K_{t}=\frac{(+\mathrm{A}-)_{w}}{(\mathrm{OAO})_{w}}=\frac{K_{b}}{K_{a}}=\frac{K_{c}}{K_{d}}
\end{aligned}
$$

In these equations, $K_{1}$ and $K_{2}$ are composite or macroscopic acid dissociation constants and $K_{t}$ is the tautomeric constant between the zwitter-ionic and neutral forms. The true partition coefficient $\left(Q^{\circ}\right)$ is given by Eq. (5). Therefore, Eq. (6) can be derived from Eq.

$$
\begin{aligned}
Q^{\circ} & =\frac{(\mathrm{OAO})_{o}}{(\mathrm{OAO})_{w}} \\
Q & =Q^{\circ} \cdot\left[\frac{1}{\frac{a}{K_{a}}+\frac{K_{c}}{a}+K_{t}+1}\right]
\end{aligned}
$$

(1), where " $a$ " is the hydrogen ion activity. In these equations the activity of the acid in each phase is assumed to be approximately the same as its concentration, when the latter is very small. When the participation of the zwitter-ionic form in the dissociation equilibrium can be excluded, the macroscopic ionization constants $K_{1}$ and $K_{2}$ correspond to $K_{a}$ and $K_{c}$, respectively, and $K_{t}$ becomes 0 . In this case, Eq. (6) can be represented by Eq. (7). As $Q^{\circ}$ is a physicochemical constant, which is independent of the concentration and $\mathrm{pH}$ of the solution but is governed by the temperature and the sort of system, Eq. (6) or

$$
Q=Q^{\circ} \cdot\left[\frac{1}{\frac{a}{K_{1}}+\frac{K_{2}}{a}+1}\right]
$$


(7) indicates that the apparent partition coefficient of an acid is constant, irrespective of the concentration at a $\mathrm{kxed} \mathrm{pH}$ value. If it is not, association or hydration of molecules would occur in either or both phases. Equation (7) also shows that $Q$ changes considerably with the $\mathrm{pH}$ of the aqueous phase, as shown in Fig. 1. The logarithmic form of Eq. (7) is represented as Eq. (8) and there is a linear relationship with a slope of +1 or -1 in the region where $\mathrm{p} K_{1} \gg \mathrm{pH}$ or $\mathrm{p} K_{2} \ll \mathrm{pH}$ (Eqs. (9) and (10)). Equation (8) also indicates that $Q$ is maxi-

$$
\begin{aligned}
& \log Q=\log Q^{\circ}-\log \left(\frac{a}{K_{1}}+\frac{K_{2}}{a}+1\right) \\
& \log Q=\mathrm{pH}-\mathrm{p} K_{1}+\log Q^{\circ} \\
& \log Q=-\mathrm{pH}+\mathrm{p} K_{2}+\log Q^{\circ}
\end{aligned}
$$

mal at the isoelectric point and when $K_{1}$ is much greater than $K_{2}, Q$ coincides with the value of $Q^{\circ}$. Therefore, if the ionization constants of an acid are known, the true partition coefficient can be calculated from Eq. (7) or Eq. (8). But determination of the ionization constants of an amphoteric acid is sometimes rather difficult because of the low solubility of the acid in water.

The true partition coefficient of an amphoteric acid, of which the ionization constant are not known, can be determined exactly applying the method of spectrophotometric determination of ionization constants developed by Ang. ${ }^{2)}$ As shown in Fig. 1, the apparent partition coefficient $Q$ has the same value at $X$ and $Y$, where the hydrogen ion activities are $a_{X}$ and $a_{Y}$, respectively. Therefore, Eqs. (11) and (12) can be used in the middle portions of the steep sections of the plots of $\log Q$ vs. $\mathrm{pH}$.

$$
\begin{aligned}
& Q=\frac{Q^{\circ}}{1+\frac{a_{X}}{K_{1}^{-}}+\frac{K_{2}}{a_{X}}} \\
& Q=\frac{Q^{\circ}}{1+\frac{a_{Y}}{K_{1}^{-}+\frac{K_{2}}{a_{Y}}}}
\end{aligned}
$$

Equations (13) and (14) are derived from Eqs. (11) and (12). These two equation indicate that there are linear relationships between $1 / Q$ and $\left(a_{X}+a_{Y}\right)$ and between $1 / Q$ and $\left(a_{X}+a_{Y}\right) / a_{X} \cdot a_{Y}$.

$$
\begin{aligned}
& \frac{1}{Q}=\frac{1}{Q^{\circ} \cdot K_{1}} \cdot\left(a_{X}+a_{Y}\right)+\frac{1}{Q^{\circ}} \\
& \frac{1}{Q}=\frac{K_{2}}{Q^{\circ}} \cdot\left(\frac{a_{X}+a_{Y}}{a_{X} \cdot a_{Y}}\right)+\frac{1}{Q^{\circ}}
\end{aligned}
$$

Thus values of $Q^{\circ}, K_{1}$ and $K_{2}$ can be readily evaluated from the slopes and intercepts on the $1 / Q$ axes of the two plots. This suggests the availability of Eqs. (13) and (14) for the determination of the ionization constants of amphoteric acids, which are only slightly soluble in water.

\section{Partitions of $p$-Aminobenzoic Acid and Sulfonamides}

Experiments on the partitions of some amphoteric acids were carried out in a system of $n$-octanol and water at $25^{\circ}$ over a wide range of $\mathrm{pH}$ values. The amphoteric acid used were $p$-aminobenzoic acid (PABA), sulfonamide (SA) and sulfamonomethoxine (SM), since they are known to take cationic, neutral and anionic forms in water and the presence of a zwitterionic form can be excluded. ${ }^{11)}$

11) I.M. Klotz and D.M. Gruen, J. Am. Chem. Soc., 67, 843 (1945). 


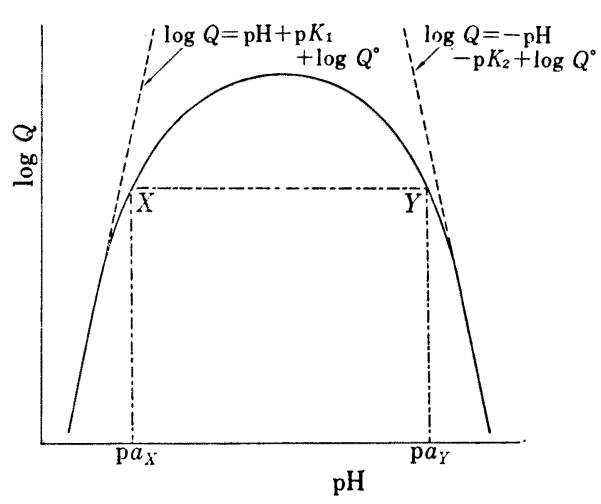

Fig. 1. Change of the Apparent Partition Coefficient $(Q)$ of an Amphoteric Acid with $\mathrm{pH}$

$Q$ has the same value at $X$ and $Y$, where the hydrogen ion activities are $\mathrm{a}_{X}$ and $\mathrm{a} Y(-\log a=\mathrm{p} a)$. The curve has two straight lines with slopes of +1 and -1 on either side.

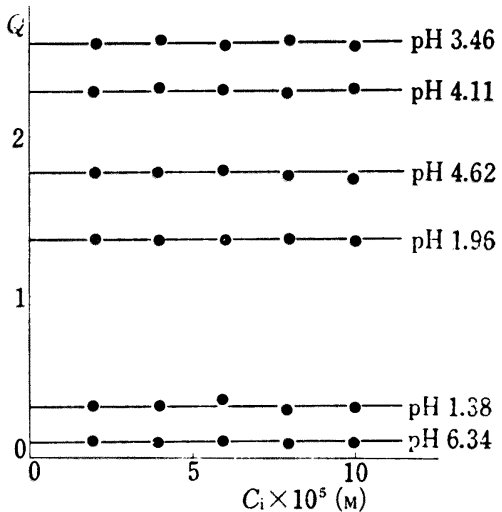

Fig. 2. Relationship between the Initial Concentration $\left(\mathrm{C}_{i}\right)$ and the Apparent Partition Coefficient $(Q)$ of $p$-Aminobenzoic Acid over a Wide $\mathrm{pH}$ Range

The apparent partition coefficients $(Q)$ were measured with various concentrations $(2 \times$ $\left.10^{-5}-10^{-4} \mathrm{M}\right)$ of these compounds. Fig. 2 shows the plot of $Q$ for PABA against its initial concentration in the aqueous phase $\left(\mathrm{C}_{i}\right)$ over a wide $\mathrm{pH}$-range. It is apparent that the value of $Q$ is constant, irrespective of $C_{i}$ at all $\mathrm{pH}$ values examined. Similar results were obtained with SA and SM in the same $C_{i}$ region.

Subsequently, the values of $Q$ are plotted against the $\mathrm{pH}$ of the aqueous phase in Figs. $3(a)$ and $4(a)$. All gave similar curves to that in Fig. 1 with two straight lines with slopes of +1 and -1 on either side, as expected from Eqs. (9) and (10). These figures clearly show that changes of the ratio of the neutral form to the total concentration of the acids in the aqueous phase $(N)$ with $\mathrm{pH}$ are similar to those of $Q$ with $\mathrm{pH}$ (Figs. $3(b)$ and $4(b)$ ). In the cases of PABA, SA, and SM, values of $N$ can be calculated from Eq. (15) using $\mathrm{p} K$ values

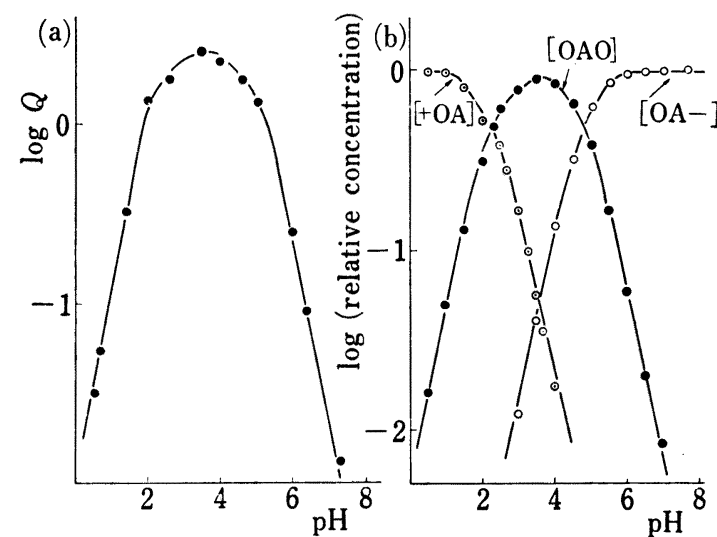

Fig. 3. (a) Variation of the Apparent Partition Coefficient $(Q)$ of $p$-Aminobenzoic Acid with $\mathrm{pH}$

(b) Relative Concentrations of Various Ionic Forms of $p$-Aminobenzoic Acid $[+\mathrm{AO}]$ : cationic form [OAO]: neutral form $[\mathrm{OA}-]$ : anionic form
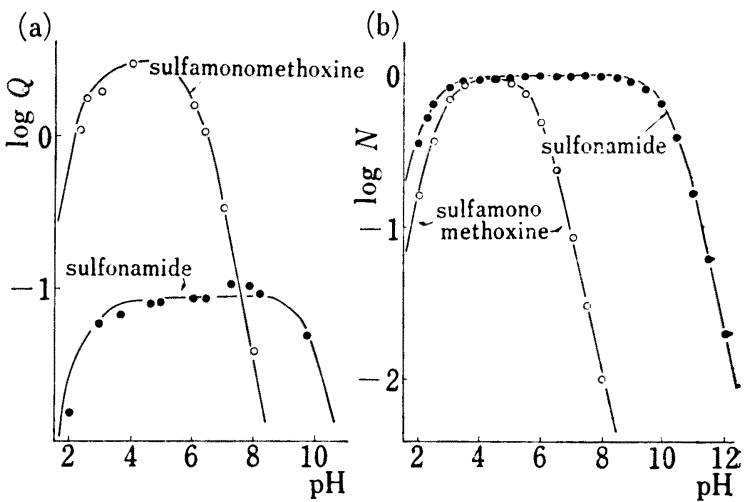

Fig. 4. (a) Variation of the Apparent Partition Coefficients $(Q)$ of Sulfonamide and Sulfamonomethoxine with $\mathrm{pH}$

(b) Relative Concentrations of the Neutral Forms $(N)$ of sulfonamide and sulfamonomethoxine 


$$
N=\frac{1}{1+\frac{a}{K_{1}}+\frac{K_{2}}{a}}
$$

determined by this partition method, as will be described later. Since the right hand of the logarithmic form of Eq. (15) is the same as the second term of the right hand of Eq. (8) and the first term of Eq. (8) is constant, it is understandable that both $Q$ and $N$ change in the same manner with $\mathrm{pH}$, if the acids are distributed between the two immiscible phases as demonstrated in Chart 1. In the cases of PABA and $\mathrm{SM}, \mathrm{Q}$ was maximal at the isoelectric point (PABA: 3.56, SM: 4.37), but in the case of SA the value of $\log Q$ was approximately constant between $\mathrm{pH} 3.72$ and $\mathrm{pH}$ 8.24. These results may be ascribed to the much greater difference between $\mathrm{p} K_{2}$ and $\mathrm{p} K_{1}$ of SA (8.07) than between those of PABA (2.49) and SM (3.25). Similar $\mathrm{pH}$-dependency of $Q$ of SA was observed in the case of sulfaguanidine distributed between iso-pentyl alcohol and water. ${ }^{12)}$ These results suggest that these amphoteric acids are distributed between $n$-octanol and the aqueous phases according to Chart 1 .

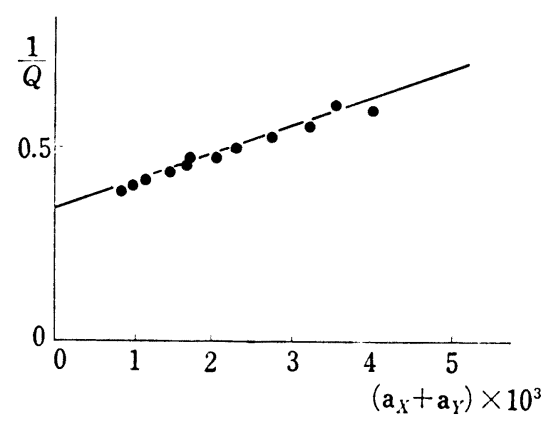

Fig. 5. Linear Relationship between $(1 / Q)$ and $\left(a_{X}+a_{Y}\right)$ of $p$-Aminobenzoic Acid

$Q$ : apparent partition coefficient $a$ : hydrogen ion activity (see Fig. 1)

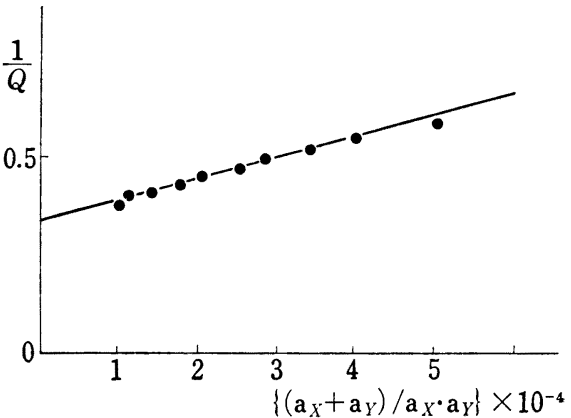

Fig. 6. Linear Relationship between $(1 / Q)$ and $\left(a_{X}+a_{Y}\right) / a_{X} \cdot a_{Y}$ of $p$-Aminobenzoic Acid

$Q$ : apparent partition coefficient $a$ : hydrogen ion activity (see Fig. 1)

From the results of partition experiments, the values of $Q^{\circ}, \mathrm{p} K_{1}$ and $\mathrm{p} K_{2}$ of these acids could be evaluated simultaneously using Eqs. (13) and (14). As an example, plots of $1 / Q$ of PABA against $\left(a_{X}+a_{Y}\right)$ and against $\left(a_{X}+a_{Y}\right) / a_{X} \cdot a_{Y}$ are shown in Figs. 5 and 6 . Linear relationships were observed and from the slopes and intercepts of the two straight lines on the $1 / Q$ axis, the values of $Q^{\circ}$ and the ionization constants were determined as described above. These values are lsited in Table I with those of 8-hydroxyquinoline (oxine) calculated from the data by Moeller and Pundsack ${ }^{13)}$ for a system of chloroform and water at $25^{\circ}$, since it was reported that presence of the zwitter-ionic form of oxine can also be excluded $\left(K_{t}=\right.$ $\left.0.04^{14)}\right)$ in its acid dissociation equilibrium.

With regard to the true partition coefficient of sulfonamides, the value of $\pi$ of SM denoted as $\left(\log Q^{\circ}{ }_{S M}-\log Q^{\circ}{ }_{S A}\right)^{15)}$ can be calculated from the results in Table I as 1.58 , which coincides well with the values of 1.55 reported by Yamazaki, et al. ${ }^{16)}$ in a system of $n$-octanol $/ \mathrm{H}_{2} \mathrm{O}$,

12) K. Kakemi, H. Sezaki, R. Konishi, T. Kimura, and A. Okita, Chem. Pharm. Bull. (Tokyo), 18, 1034 (1970).

13) T. Moeller and F.L. Pundsack, J. Am. Chem. Soc., 75, 2258 (1953).

14) S.F. Mason, J. Chem. Soc., 1958, 674.

15) T. Fujita, J. Iwasa, and C. Hansch, J. Am. Chem. Soc., 86, 5175 (1964).

16) M. Yamazaki, N. Kakeya, T. Morishita, A. Kamada, and M. Aoki, Chem. Pharm. Bull. (Tokyo), 18, 708 (1970). 
TABLE I. Values of True Partition Coefficients $\left(Q^{\circ}\right)$ and Inonization Constants $(K)$ of Some Amphoteric Acids

\begin{tabular}{|c|c|c|c|c|c|}
\hline \multirow{2}{*}{ Acid } & \multirow{2}{*}{$\log Q^{\circ a)}$} & \multicolumn{2}{|c|}{$\mathrm{p} K_{1}$} & \multicolumn{2}{|c|}{$\mathrm{p} K_{2}$} \\
\hline & & Cal. & Other data $\left.{ }^{b}\right)$ & Cal. & Other data $\left.{ }^{b}\right)$ \\
\hline p-Aminobenzoic Acid & 0.46 & 2.31 & $2.36-2.50$ & 4.80 & $4.80-4.85$ \\
\hline Sulfonamide & -1.05 & 2.24 & $2.06-2.36$ & 10.31 & $10.00-10.70$ \\
\hline Sulfamonomethoxine & 0.53 & 2.74 & - & 5.99 & 5.94 \\
\hline 8-Hydroxyquinoline ${ }^{c}$ ) & 2.56 & 4.99 & $5.00-5.05$ & 9.90 & $9.66-9.85$ \\
\hline
\end{tabular}

a) $\log Q^{\circ}$ were determined in a system of $n$-octanol $/ \mathrm{H}_{2} \mathrm{O}$ at $25^{\circ}$, except in the case of 8 -hydroxyquinoline.

b) Cited from the data of the following literatures. They were determined by potentiometric and spectrophotometric methods. I.M. Klotz and D.M. Gruen, J. Am. Chem. Soc., 67, 843 (1945); P.H. Bell and R.O. Jr. Roblin, ibid., 64, 2905 (1942); P.O. Lumme, Suomen Kemistilehti, 30B, 176 (1957); A. Albert and R. Goldacre, Nature, 149, 245 (1942); J.H. Gorvin, J. Chem. Soc., 1949, 3304; A.V. Willi, Helv. Chim. Acta, 39, 46 (1956); J. Rieder, Arzneimittel Forsch., 13, 84 (1963); D. Dyrssen, Svensk Kem. Tid., 64, 213 (1952).

c) Calculated from the data of Moeller and Pundsack. ${ }^{13)} \quad Q^{\circ}$ was determined in a system of chloroform/ $\mathrm{H}_{2} \mathrm{O}$.

and of 1.49 reported by Fujita and $\mathrm{Hansch}^{17)}$ in a system of isobutanol/ $\mathrm{H}_{2} \mathrm{O}$ using the experimental data of Rieder. ${ }^{18)}$ The value of $\pi$ in an $n$-octanol $/ \mathrm{H}_{2} \mathrm{O}$ system is known to agree well with that in an isobutanol $/ \mathrm{H}_{2} \mathrm{O}$ system. ${ }^{19}$ ) Since $\pi$ is an additive constant, when strong inter- and intra-molecular interactions are absent, $\log Q^{\circ}$ can be estimated as the sum of $\pi$ for each functional group in the molecule. ${ }^{20}$ Therefore, the value of $\log Q^{\circ}$ of $\mathrm{PABA}$ can be represented as follows:

$$
\log Q^{\circ}{ }_{\mathrm{PABA}}=\Pi_{\mathrm{COOH}}+\Pi_{\mathrm{NH}_{2}}+\log Q^{\circ}{ }_{\text {benzene }}
$$

Taking the value of -0.28 for $\pi_{\mathrm{COOH}}{ }^{15)}-0.46$ for $\pi_{\mathrm{NH}_{2}}{ }^{15)}$ and 2.13 for $\log Q^{\circ}{ }_{\text {benzene }}{ }^{20)}$ the calculated value of $\log Q_{\text {PABA }}^{\circ}$ becomes 1.39. This value is approximately the same as the experimental value of 1.51. However, the value of $\pi_{\mathrm{NH}_{2}}$ ranged from -1.63 to -0.46 for the compound examined, ${ }^{15}$ ) so it is not clear whether there is some discrepacy between experimental and calculated values of the true partition coefficient of PABA or not. The scattered value of $\pi_{\mathrm{NH}_{2}}$ reported by Fujita et al. ${ }^{15)}$ suggests the presence of strong solutesolvent interaction in the compound of having $-\mathrm{NH}_{2}$ groups.

In Table I, the ionization constants of the acids obtained by other methods are also listed. It is clear that the values of $\mathrm{p} K_{1}$ snd $\mathrm{p} K_{2}$ obtained by this method were in good agreement with those obtained by other authors using other methods. These results suggest the availability of this method for determination of the ionization constants of amphoteric acids.

However, when the partition of the zwitter-ionic form can not be excluded or the $K_{t}$ value becomes greater, as observed with cysteamine, ${ }^{21)}$ the true partition coefficient can not be determined exactly by this partition method alone, since Eq. (7) is not available for this purpose and instead of Eqs. (13) and (14) following equations must be used:

$$
\begin{aligned}
& \frac{1}{Q}=\frac{1}{Q^{\circ} \cdot K_{a}} \cdot\left(a_{X}+a_{Y}\right)+\frac{K_{t}+1}{Q^{\circ}} \\
& \frac{1}{Q}=\frac{K_{c}}{Q^{\circ}} \cdot\left(\frac{a_{X}+a_{Y}}{a_{X} \cdot a_{Y}}\right)+\frac{K_{t}+1}{Q^{\circ}}
\end{aligned}
$$

17) T. Fujita and C. Hansch, J. Med. Chem., 10, 991 (1967).

18) J. Rieder, Arzneimittel-Forsch., 13, 84 (1963).

19) W. Scholtan, Arzneimittel-Forsch., 18, 505 (1968).

20) E. Miller and C. Hansch, J. Pharm. Sci., 56, 92 (1967).

21) H. Tanaka, H. Sakurai, and A. Yokoyama, Chem. Pharm. Bull. (Tokyo), 18, 1015 (1970). 
Furthermore, in the cases of such acids as glycine ${ }^{22)}$ and selenoxine, ${ }^{23)}$ of which $K_{t}$ values are very large (the presence of the unionized form of the acids is negligible), Eq. (19) can be derived from Eq. (6). Eq. (19) indicated that the change of $Q$ with $\mathrm{pH}$ is similar to those of PABA

$$
Q=\frac{Q^{\circ}}{K_{t}} \cdot\left(\frac{1}{-\frac{a}{K_{1}^{-}}+\frac{K_{2}}{a}+1}\right)
$$

and sulfonamides, but the true partition coefficient can not be determined, if $K_{t}$ is not known. The partition behavior in this case will be reported in the succeeding paper.

\section{Experimental}

Reagents_-Sulfonamides (Daiichi Seiyaku Co., Ltd.) and p-aminobenzoic acid (Wako Pure Chemical Industries Ltd.) of guaranteed grade were used without further purification.

Determination of Partition Coefficients — Aqueous solutions of $2.0 \times 10^{-5}-10^{-4} \mathrm{M}$ acid at appropriate $\mathrm{pH}$ values were shaken with $n$-octanol in a water bath at $25 \pm 0.1^{\circ}$. After equilibrium had been attained, the concentration of acid was determined spectrophotometrically at an appropriate wavelength. The apparent partition coefficient was calculated from Eq. (20), where $C$ and $V$ denote the concentrations and

$$
\begin{aligned}
Q & =\mathrm{C}_{o} / \mathrm{C}_{w} \\
& =\frac{\left(C_{i}-C_{w}\right)}{C_{w}} \cdot\left(\frac{V_{w}}{V_{o}}\right)
\end{aligned}
$$

volumes of the aqueous (subscript $w)$ and $n$-octanol (subscript $o$ ) phases, respectively. $\left(\mathrm{V}_{w} / \mathrm{V}_{o}\right)$ changed with $\mathrm{pH}$ so that the condition $1<\left(\mathrm{C}_{i}-\mathrm{C}_{w}\right) / \mathrm{C}_{\boldsymbol{w}}<10$ was $\mathrm{kept}$, and each phase was previously equilibrated with the other.

Acknowledgement The author is indebted to Miss M. Negayama for her expert technical assistance.

22) J.T. Edsall and J. Wyman, "Biophysical Chemistry," Vol. 1, Academic Press, New York, 1958, p. 477.

23) E. Sekido, Q. Fernando, and H. Freiser, Anal. Chem., 36, 1768 (1964). 